\title{
Dermatological high-dose-rate brachytherapy for the treatment of basal and squamous cell carcinoma
}

\author{
A. F. Sedda, G. Rossi, C. Cipriani,* A. M. Carrozzot and P. Donati‡ \\ Physical Technologies and New Materials (FIM), Italian Agency for New Technologies, Energy and Environment (ENEA) Casaccia, Rome, Italy; *Nuclear Medicine \\ Department, S. Eugenio Hospital, Rome, Italy; †Dermatology Department, Tor Vergata University, Rome, Italy; and ¥S. Gallicano Hospital, IFO, Rome, Italy \\ doi:10.1111/j.1365-2230.2008.02852.x
}

\section{Summary}

\begin{abstract}
Background. Basal cell carcinoma (BCC) and squamous cell carcinoma (SCC) are among the most common cancers in humans. Various therapies are currently being used to treat these tumours including surgery, topical treatments and radiotherapy. We describe a new treatment for BCC and SCC. This consists of superficial radiotherapy, using synthetic resin containing a radioactive $\beta$-emitting isotope. The resin is applied to the lesion to perform a selective $\beta$-irradiation brachytherapy treatment.

Methods. In total, 53 patients with histologically confirmed diagnosis of BCC and of SCC were enrolled for the treatment.

Results. In all treated cases, an apparent clinical remission occurred in approximately 3 months, and complete healing was obtained in 100\% of the treated patients; in $82 \%$ of the cases, this occurred after a single application. No disfiguring scars or any side-effects were seen. After a follow-up of 20-72 months (mean 51 months), no clinical relapses were observed in the treated patients. Histological examination confirmed complete tumour regression.

Conclusion. The results indicated that brachytherapy is an effective treatment for BCC and SCC.
\end{abstract}

\section{Introduction}

Skin cancer is one of the most common forms of cancer, and basal cell carcinoma (BCC) is the most common cancer in white populations. ${ }^{1}$ Squamous cell carcinoma (SCC) is the second most common form of skin cancer, and in European countries its annual rate of incidence is about 25/100 000 in the population. SCC is a more aggressive tumour than BCC, may metastasize to regional lymph nodes and is often locally recurrent. ${ }^{2}$

For both tumours, standard treatments such as curettage and cautery, surgery (including Mohs' surgery), cryosurgery, ${ }^{3,4}$ topical chemotherapy, imiquimod and photodynamic therapy ${ }^{5}$ are often proposed to

Correspondence: Dr Antioco Franco Sedda, FIM, ENEA-Casaccia s.p. Anguillarese 301, 00123 Rome, Italy.

E-mail: antioco.sedda@casaccia.enea.it

Conflict of interest: none declared.

Accepted for publication 25 January 2008 patients. Radiotherapy, using low-energy X-rays $(\leq 90 \mathrm{kVs})$ or electrons, and interstitial brachytherapy with $\gamma$-ray emitting sources placed across the tumour volume, have both been used in clinical practice. ${ }^{6}$

In this paper, we present a new treatment for BCC and of SCC. This consists of superficial radiotherapy, characterized by the use of a specially formulated, inert, synthetic resin containing radioactive $\beta$-emitting isotopes, which is able to adapt to skin surfaces without spreading beyond the tumour area, allowing accurate dose distribution and sparing of healthy tissue.

The treatment has been used in a large variety of BCC and SCC forms, from very large tumours to relapsing or recurrent forms, to multifocal lesions.

\section{Methods}

The treatments were routinely performed in the Nuclear Medicine Department of S. Eugenio Hospital. The method of preparing radioactive resin has previously been reported. ${ }^{7,8}$ Briefly, carrier-free ${ }^{188}$ Re (perrhenate) 
was obtained from a ${ }^{188} \mathrm{~W} /{ }^{188} \mathrm{Re}$ generator (Oak Ridge National Laboratory, Oak Ridge, TN, USA) by elution with saline. A sterile nanocolloid $(200-800 \mathrm{~nm})$ of $\mathrm{Re}_{2} \mathrm{~S}_{7}$ was obtained by reaction of perrhenate with hydrogen sulphide. The nanocolloid was thoroughly mixed with a synthetic acrylic resin (Meyer, Milan, Italy) to obtain homogeneous distribution of the radioactive nanocolloid.

The skin to be treated was protected by a thin layer of a protective cream (Tueor; SOFAR, Milan, Italy), and the radioactive resin was applied on top of this cream layer. After a few minutes, the resin solidified without appreciable shrinkage; the radioactive mould was kept on the lesions for the time necessary to impart the precalculated dose distribution.

The thickness of the resin and cream was accurately measured, in order to account for $\beta$-radiation absorption and self-absorption effects. For each application, the dose distribution depends on the initial radioactivity, the isotope emission energy, the surface of the lesion and the contact time. The applied radioactivity was measured using a dose calibrator (Model 4045; RadCal, Monrovia, CA, USA). A Perspex screen $10 \mathrm{~mm}$ thick was sufficient to protect the physician from $\beta$-radiation during the phase of application (1-3 $\mathrm{min}$ ) to the patient. During the irradiation, typically lasting from $15 \mathrm{~min}$ to 2 hours, the patient was kept isolated; after the required irradiation time, a radioactivity test was performed on the treated lesions, which always showed a total absence of any measurable contamination.

\section{Dosimetry}

The ${ }^{188}$ Re isotope is a mixed $\beta-\gamma$ emitter, with a half-life of 16.98 hours; the $\beta$-particles have a maximum energy of $2.12 \mathrm{MeV}$ and a mean energy of $764 \mathrm{keV}$, therapeutically effective only at short ranges. A $\gamma$-ray component of $155 \mathrm{keV}$ accounts for $15 \%$ of the radiation intensity, and allows excellent control of any possible radioactive contamination.

The dose distribution of $\beta$ particles in human tissue is described by complex multiexponential functions. ${ }^{9-12}$ Vinkyer et al., ${ }^{13}$ and Sedda et al., ${ }^{14}$ proposed some modifications of the classic formulas, in order to increase the accuracy of dose distribution at distances comparable to the maximum range of the $\beta$ particles.

For each application, a calculation of the dosedistribution curve imparted to the skin was performed by a point source algorithm, but in selected cases an intercomparison of different calculation methods was performed, by comparing the results obtained using EGS4 Monte Carlo software, a point source numerical integration algorithm, and $a b$ initio integration calculations of point sources of $\beta$ rays. An agreement of the dose-distribution curves obtained from the different models within $\pm 5 \%$ was found.

\section{Treatments}

In total, 53 patients (29 men, 24 women) with histologically confirmed diagnosis of BCC (37 patients) or SCC (16 patients) were enrolled. These were patients in whom a relapse of the tumour had occurred, or for whom surgery was considered impossible or aesthetically unacceptable. Nine patients had multiple BCC lesions and three patients had multiple SCC lesions. Within the study group, $70 \%$ of the tumours were present on the head or neck, $22 \%$ on the upper and lower limbs, and $8 \%$ on the trunk and back. Most of the superficial BCCs on the head were located on the nose and temporal area. Tumours of the nodular or infiltrative type made up $32 \%$ of the total. Further details on patients are shown in Table 1.

A thorough clinical and dermoscopical examination of the lesional area was performed, and histological information was used to evaluate the mean depth of tumours and the limit of the histologically healthy tissue of the lesions. High-resolution digital photographs of the tumours were collected before and at various intervals after the treatment to evaluate and document clinical healing.

For each patient and for each lesion, the dosedistribution curve was always calculated, using a point source integration program. Mean doses of 40-60 Gy were given, to depths of 300-600 $\mu \mathrm{m}$ from the epidermis, depending on histological indications. Some typical examples of dose distribution curves are reported in Figure 1. The activity used for each treatment ranged

Table 1 Overview of patient data and treatments.

\begin{tabular}{lcc}
\hline Tumour & BCC & SCC \\
\hline Treatments (no. of patients) & 32 & 11 \\
1 & 4 & 4 \\
2 & 1 & 1 \\
3 & $7.04 \pm 8.9$ & $14.6 \pm 10.6$ \\
Mean \pm SD area ( $\left.\mathrm{cm}^{2}\right)$ & & \\
Follow-up (no. of patients) & 6 & 3 \\
1-2 years & 14 & 4 \\
3-4 years & 12 & 4 \\
$\geq 5$ years or more & & \\
Previous surgical treatment $(n)$ & 20 & 8 \\
$\quad$ No & 12 & 3 \\
$\quad$ Yes & & \\
\hline
\end{tabular}




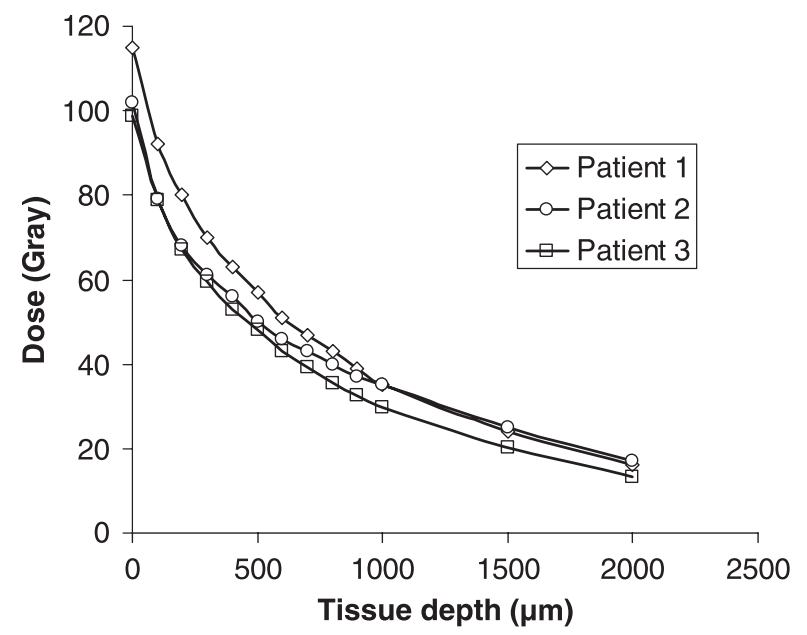

Figure 1 Example of ${ }^{188}$ Re dose distribution curves in three different patients.

from $40 \mathrm{MB}$ of ${ }^{188} \mathrm{Req}$ in the smaller lesions up to about $2000 \mathrm{MBq}$ in larger lesions. The required application times varied from a few minutes to 2 hours, depending on the applied radioactivity, the size of the lesion, and the calculated dose-distribution curve.

\section{Results}

Immediately after the irradiation, mild erythema was visible in the irradiated area, which completely disappeared 2-7 days after the treatment. Bleeding was often present in large lesions; this ceased 10-30 days after treatment, a scab was formed, and the lesion gradually healed. Most of the patients only needed a single treatment; in some cases two or three treatments were required for complete healing (Table 1). The number of treatments required for healing is apparently related to the thickness of the lesion; thicker tumours generally required two or even three treatments.

In some cases, clear neoangiogenic development was seen in the dermoscopic examination of the tumour area, especially in SCC cases, which gradually disappeared after the treatment.

In all treated cases, apparent complete clinical remission occurred after 3-5 months; in all the cases in which a second or even a third treatment was performed, the ulcerations rapidly disappeared after the first treatment, and the tumour residue appeared as a hard nodule. After successive treatments, a gradual flattening and softening of the lesion was observed, ending with complete healing (some examples of clinical results are reported in Figs 2-4).
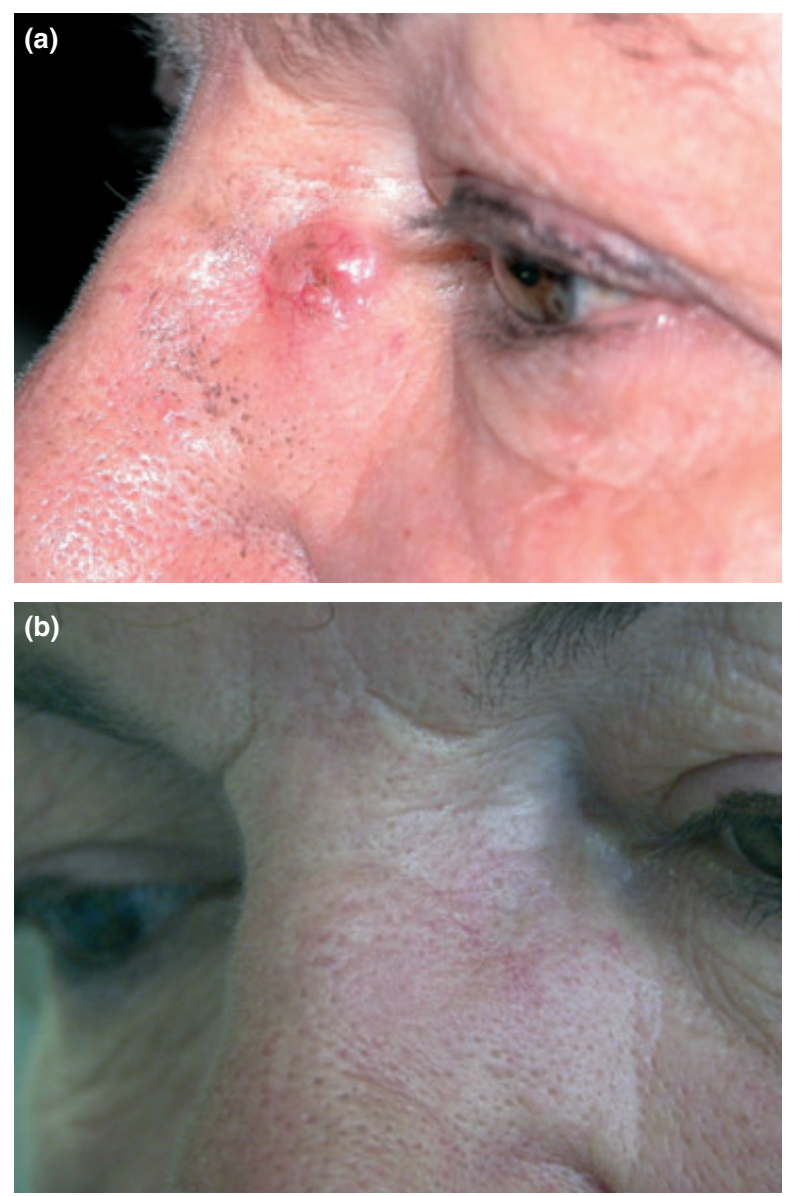

Figure 2 Basal cell carcinoma on the nose (a) before and (b) 120 days after a single treatment. Estimated imparted dose was 50 Gy at $450 \mu \mathrm{m}$.

After a follow-up of 20-72 months (mean 51) complete healing was seen in $100 \%$ of the treated cases; in $82 \%$ of the patients, this occurred after a single application. In the remaining cases, a maximum of three treatments was used (Table 1). No disfiguring scarring, pain or side-effects were seen. After the treatment, the patients were discharged home without any further prescriptions.

Follow-up included a control visit at 1 week, 3-4 weeks, 3 months, 6 months, and finally 1 visit/ year. For each visit, photographic documentation of the lesion appearance was always collected. After a follow up of 20-72 months, no systemic nor topical side-effects had occurred, and no relapses were seen.

Moreover, treatment was successful not only for superficial tumours, but also for nodular, infiltrative, ulcerating and relapsing tumours, which showed relatively rapid and complete healing. Histological 

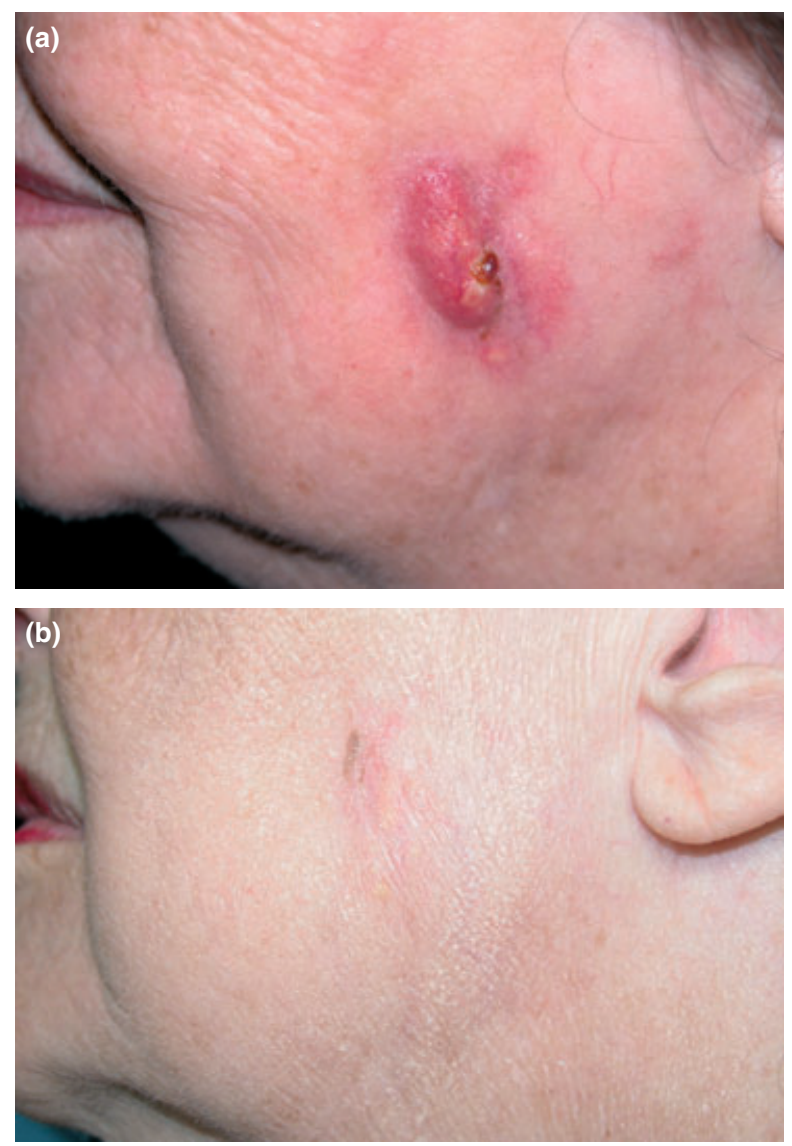

Figure 3 Squamous cell carcinoma on the cheek (a) before and (b) 60 days after a single treatment. Estimated imparted dose was 50 Gy at $600 \mu \mathrm{m}$.

examination performed after the treatment (for about $60 \%$ of the patients) confirmed complete tumour regression.

\section{Discussion}

There is general agreement that surgery is the treatment of choice for BCC and SCC. Alternative treatments offer cost and clinical advantages in selected cases, such as for tumours located in high-risk areas or difficult sites (nose, ears, eyelids), or for patients with a large number of lesions, and older, infirm, or otherwise inoperable patients.

Irradiation of BCC by photons has been used in clinical practice in selected cases; high cure rates have been obtained, with values almost comparable to Mohs' micrographic surgery. ${ }^{1,5}$ However, classic photon radiotherapy faces the problem of relatively inaccurate irradiation of tumour margins, due to the lack of
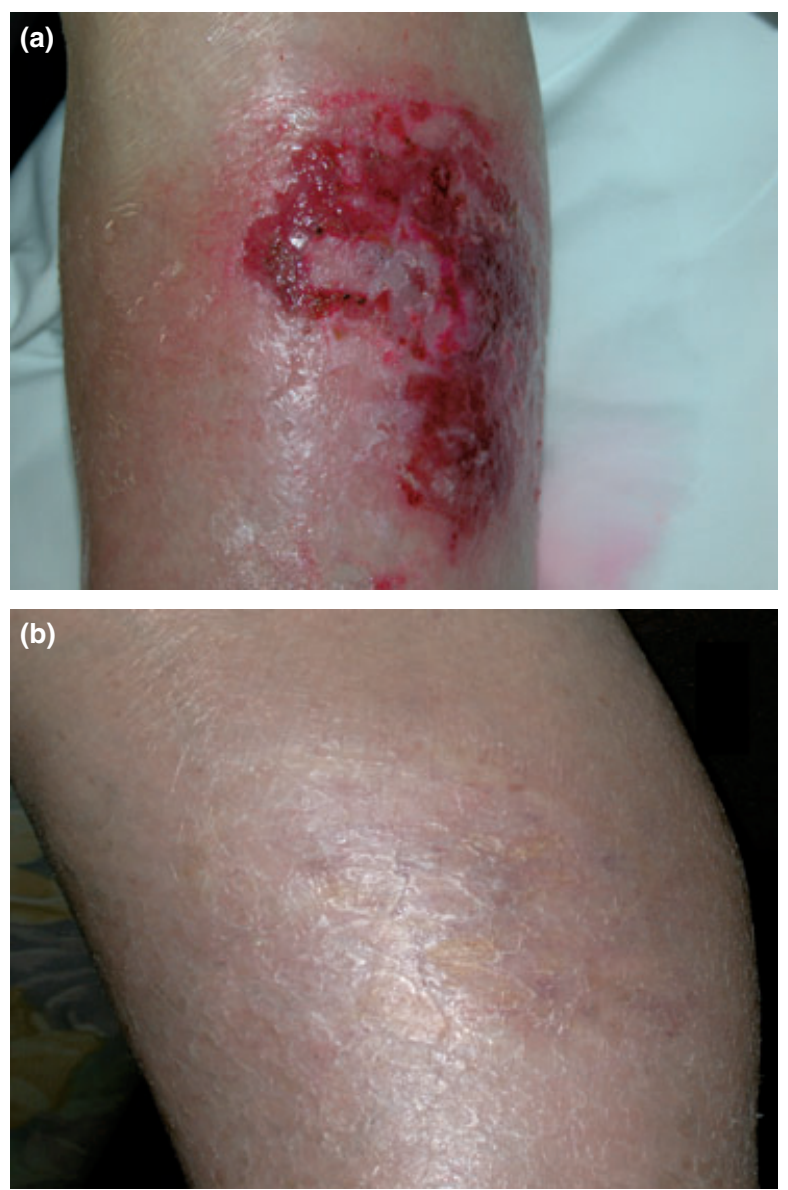

Figure 4 Squamous cell carcinoma on the leg (a) before and (b) 120 days after a single treatment. Estimated imparted dose was 50 Gy at $500 \mu \mathrm{m}$.

individually moulded radiation collimators, and can be harmful to adjacent organs, due to the penetrating nature of $\gamma$ photons or X-rays. Classic $\gamma$ brachytherapy requires complex software programs for treatment planning and calculation of dose distribution to obtain an individually adjusted dose distribution, but the dose distribution remains far from ideal, due to the penetrating nature of the photons, and a large number of sessions (30-40) are usually required. ${ }^{6}$

The use of $\beta$ radiation embedded in a tailor-made irradiation mould, such as the technique we describe here, appears able to override the drawbacks of classic radiotherapy. In fact, not only can the margin of irradiation be easily controlled by the present technique, but the dose-distribution curve obtained by beta particles (Fig. 1) almost follows the typical distribution of tumour invasion in dermal tissue, administering the therapeutic dose only at the required depths, without 
unnecessary dose deposition in the subdermal tissue, independently of the complexity of the lesion shape or the number of lesions.

It must be noted that in our treatments the mean dose distribution curve (Fig. 1) showed a clear reduction in dose from a nominal value of 100-120 Gy at the epidermis to $<20$ Gy at a depth of only $2 \mathrm{~mm}$, a depth at which invasion from tumour cells is usually present.

Although it is difficult to reconcile such superficial treatment with the excellent clinical results we obtained, it must be noted that single-fraction radiotherapy has been used for the treatment of small superficial BCCs and SCCs in $>800$ patients, obtaining an overall disease-free rate at 5 years of $84 \%$; the optimum applied dose for such a lesion on a flat surface was $20 \mathrm{~Gy},{ }^{15,16}$ which suggests fairly high radiosensitivity for these class of tumours.

Animal studies also indicate that ionizing radiation may exhibit immunomodulatory properties by enhancing antitumour immune response; radiation upregulates the expression of various cytokines (e.g. interleukins $1 \mathrm{a}$, $1 \mathrm{~b}, 2$ and 12, and tumour necrosis factor- $\alpha$ and $-\beta$ ) in tumour, stromal and infiltrating cells. ${ }^{17}$

It has recently been recognized that doses of radiation lower than or equal to those that cause direct cytolysis may alter the phenotype of target tissue, by upregulating gene products that may make tumour cells more susceptible to T-cell-mediated immune attack. ${ }^{18}$ Thus a synergistic mechanism may be present in the clinical and histological healing of tumour lesions apparently thicker than the therapeutic range of the $\beta$ particles.

Apart from the underlying mechanism, the clinical results obtained with the technique we describe were satisfactory for the whole cohort of treated patients, in spite of the relatively small observation group. The proposed technique should be considered a new clinical treatment, and an alternative to both surgical and medical treatments. Its main advantage lies in the usefulness in all types of BCC and SCC, without restriction by site, dimension, clinical or histological type, or the patient's clinical situation. It is a rapid treatment, usually performed in a single treatment session, without discomfort for the patient, and offers complete aesthetic restitutio ad integrum.

\section{References}

1 American Cancer Society. Cancer Facts and Figures. ACS: Atlanta. http://www.cancer.org/downloads/STT/2008 CAFFfinalsecured.pdf.
2 Lee K, McKean ME, McGregor IA. Metastatic patterns of squamous carcinoma in the parotid lymph nodes. Br J Plast Surg 1985; 38: 6-10.

3 Margoob AA. Basal and squamous cell carcinomas: what every primary care physician should know. Postgrad Med 1997; 102: 139-59.

4 Martinez JC, Otley CC. The management of melanoma and non-melanoma skin cancer: a review for the primary care physician. Mayo Clinic Proc 2001; 7: 63.

5 Peng Q, Warloe T, Berg K et al. 5-aminolevulinic acidbased photodynamic theraphy. clinical research and future challenges. Cancer 1997; 79: 2282-308.

6 Guix B, Finestres F, Tello JI et al. Treatment of skin carcinomas of the face by high-dose-rate brachytherapy and custom-made surface molds. Int J Radiat Oncol Biol Phys 2000; 4: 95-102.

7 Sedda AF, Rossi G, Cipriani C. Beta emitter multilayer for the dermatological brachytherapy of cutaneous tumours. Eur J Nucl Med 2003; 3: S343.

8 Sedda AF, Rossi G, Carrozzo AM et al. Superficial brachytherapy with beta emitting isotopes for the treatment of basal cell carcinoma. J Invest Dermatol 2006; 126: S37.

9 Loevinger R. The dosimetry of beta sources in tissue. The point source function. Radiology 1956; 66: 55-62.

10 Cross WG. Tables of Beta Dose Distributions. Report AECL2793. Chalk River, ON: Atomic Energy of Canada Ltd., 1967.

11 Berger MJ. Complete distribution of absorbed dose around point sources of electrons and beta particles in water and other media. MJ MIRD pamphlet no. 7. J Nucl Med 1971; 12 (Suppl.): 1-23.

12 Valley JF, Kurshelevsky AP, Lerch P. A method for calculation of beta ray dose. Health Phys 1974; 26: 295-300.

13 Vynckier S, Wambersie A. Dosimetry of beta sources in radiotherapy: absorbed distributions around plane sorces. Radiat Prot Dosimetry 1986; 14: 169-73.

14 Sedda AF, Rossi G, Desantis M et al. DOBBIN (Distribution Optimization for Beta Brachytherapy Intratumour): a program for geometrical optimization of beta brachytherapy interstitial administration. Quart J Nucl Med 2000; 2: 79.

15 Wilder RB, Kittelson JM, Shimm DS. Basal cell carcinoma treated with radiation therapy. Cancer 1991; 68: 2134-7.

16 Chan S, Dhadda AS, Swindell R. Single fraction radiotherapy for small superficial carcinoma of the skin. Clin Oncol (R Coll Radiol) 2007; 19: 256-9.

17 Friedman EJ. Immune modulation by ionizing radiation and its implications for cancer immunotherapy. Curr Pharm Des 2002; 8: 1765-80.

18 Garnett CT, Palena C, Chakraborty M et al. Sublethal irradiation of human tumor cells modulates phenotype resulting in enhanced killing by cytotoxic T lymphocytes. Cancer Res 2004; 64: 7985-94. 